
\title{
is Research Suare \\ Adverse Childhood Experiences and Implications of Perceived Stress, Anxiety, and Cortisol Among Women in Pakistan
}

Naira Ikram ( $\nabla$ ni22@duke.edu )

Duke University https://orcid.org/0000-0003-0134-7228

Allison Frost

UNC Gillings

Katherine LeMasters

UNC Gillings

Ashley Hagaman

Yale

Victoria Baranov

University of Melbourne

John Gallis

Duke

Siham Sikander

Human Development Research Foundation

Elissa Scherer

RTI

Joanna Maselko

UNC Gillings

\section{Research Article}

Keywords: adverse childhood experiences, stress, anxiety, cortisol, Pakistan

Posted Date: April 8th, 2021

DOI: https://doi.org/10.21203/rs.3.rs-403429/v1

License: (9) (i) This work is licensed under a Creative Commons Attribution 4.0 International License. Read Full License 


\section{Abstract}

Background: Adverse Childhood Experiences (ACEs) are linked to poor maternal mental health. By disrupting stress regulation systems, ACEs are hypothesized to impact perceived stress, anxiety, and cortisol. This study explores the associations of ACEs with different manifestations of stress.

Methods: Participants were part of the Bachpan study, a longitudinal birth cohort in rural Pakistan. Data were collected at the 36-month postpartum wave. ACEs were captured retrospectively using an adapted version of the ACE International Questionaire, and represented in the following ways: as a continuous variable, binary indicator, categoric levels, and subdomains (neglect, home violence, family psychological distress, community violence). Outcomes included: perceived stress ( $\mathrm{N}=889)$ measured with the Cohen Perceived Stress Scale (PSS), anxiety $(\mathrm{N}=623)$ measured with the Generalized Anixety Disorder-7 scale (GAD-7), and hair-derived cortisol ( $N=90)$. Multivariable linear mixed models estimated associations between ACEs and the outcome variables.

Results: All models featured positive associations between ACE items and PSS. Both the continuous total ACE score $(B=0.4 ; 95 \% C l=0.0,0.8)$ and the presence of any $A C E s(B=1.0 ; 95 \% C l=-1.0,0.3)$ were associated with higher anxiety symptoms on the $G A D-7$. Home violence $(B=6.7 ; 95 \% \mathrm{Cl}=2.7,10.8)$ and community violence $(B=7.5 ; 95 \% \mathrm{Cl}=1.4,13.6)$ were associated with increased hair cortisol.

Conclusions: All four ACE domains were associated with elevated levels of perceived stress, anxiety, and cortisol, with varying precision and strength of estimates, indicating that the type of ACE has a differential impact. This study disentangled adversity to understand the impact of specific adverse events on hypothalamic pituitary adrenal (HPA) axis functioning and mental health conditions.

\section{Background}

The prevalence of Adverse Childhood Experiences (ACEs) is extremely high, with population estimates ranging from 50\%-70\% (Felitti et al., 1998; Ramiro, 2010). A robust literature links ACEs with compromised physical health, mental health, and overall functioning in adulthood (1-4). Those exposed to ACEs are less likely to obtain high levels of education, leading to financial insecurity, lower socioeconomic status, and a lack of psychosocial resources (4). The compounding nature of adversity is especially impactful in low- and middle-income countries (LMICs), where rates of ACEs are high and other stressors, such as poverty, may lead to and co-occur with ACEs $(5,6)$. The impact of ACEs on maternal mental health is of particular concern as maternal mental health may function as a mediator between maternal ACEs and child mental health, indicating intergenerational trauma (7). Informed by life course theory's linkage of adult disease risk to psychosocial exposures stemming from childhood, understanding the associations between ACEs and adult health may offer important insights into preventing compounding health issues throughout life (8).

Although the overall literature linking ACEs to mental health is robust, several key questions are not well understood. The first is to what extent examining the unique effects of different types of ACEs is important for elucidating how early experiences impact adult functioning. Much of past work has focused on specific ACEs (e.g., sexual abuse) or the cumulative effect of ACEs, and often, domain-specific (e.g., abuse, neglect, household dysfunction) results have either not been reported or have been ambiguous (9-11). Nonetheless, multiple researchers have pointed to the utility of separating ACEs into distinct domains. For instance, Negriff (2020) found that in the United States, experiencing neglect, but not physical abuse, predicted anxiety symptoms in 
adolescence and ACEs related to child maltreatment were more predictive of mental health outcomes than ACEs related to household dysfunction (12). In our team's prior work with a cohort of women in Pakistan at 36 months postpartum, we have found that exposure to community violence was much more strongly correlated with maternal depression than other domains such as neglect (13). A key potential driver of different findings is the diversity of social, economic, and cultural contexts in which the ACEs take place. Hence, studies from multiple geographic regions and population groups make important contributions towards better understanding of how different ACEs may differentially impact mental health.

A second area of inquiry focuses on the biological stress-related pathways linking ACEs to various health outcomes. Specifically, there is evidence that ACEs impact stress regulation systems, including the Hypothalamic Pituitary Adrenal (HPA) axis and its end product of cortisol (14-16). ACEs are associated with both hyperactivation and hypoactivation of the HPA axis, leading to elevated or reduced cortisol levels, respectively (17-19). The majority of this research has also been conducted in high income settings. The interplay among ACEs, adult stress and anxiety, and adult stress system functioning is not well understood in LMICs and has not been explored in South Asia specifically $(4,20,21)$. HPA axis hormones extracted from hair samples capture chronic stress, which is an important mediator between early life experiences and later mental health (22).

In the current study, we examine how ACEs are associated with multiple dimensions of stress later in life including anxiety, perceived stress, and hair derived cortisol in a sample of mothers living in rural Pakistan. We investigate ACEs, and their specific domains, in a LMIC setting because they have been underexplored in this region. For example, while the majority of past studies on these topics sample well-educated, urban populations, the majority of Pakistani women have spent their life in rural areas, which is tied to unique stressors, such as food insecurity, low education and literacy of women, and transportation barriers, compared to urban settings many past studies are set in (23-26).

\section{Methods}

\section{Study Design and Participants}

The data in the present analysis come from mothers of the Bachpan cohort, a longitudinal birth cohort of motherchild dyads in rural Pakistan north of the Punjab Province (Sikander et al., 2015). One of the purposes of the Bachpan cohort was to evaluate the impact of a peer-delivered, community-based perinatal depression intervention, embedded within the cohort, on maternal mental health and child development (27). Pregnant women from 40 village clusters were screened for depression and invited to participate in the study. Twenty of the 40 village clusters were randomized to receive the perinatal depression intervention delivered by lay peers and 20 clusters received enhanced usual care. In addition, an equal number of non-depressed pregnant women were recruited and followed in parallel as part of the Bachpan cohort. Women were assessed at baseline (during their third trimester of pregnancy) and at an additional five time points: three, six, 12, 24, and 36 months postpartum. At the 36-month assessment, participants completed questionnaires assessing ACEs, anxiety, and perceived stress. In addition, a subsample of participants at the 12 month interview, were asked to provide hair for cortisol assay. Of the 1,154 women who were included at baseline, 889 participated in the 36 month questionnaire, providing information on ACEs and perceived stress. Due to a delay in administering the anxiety assessment, 623 participants provided information on anxiety. We approached a randomly-selected subsample of 107 women for 
hair cortisol collection, and of those, 93 provided valid samples. Finally, 90 mothers provided both hair cortisol and ACEs measurement.

\section{Measures}

Adverse childhood experiences. ACEs were assessed at the 36 month data collection period using an adapted 12item ACE International Questionnaire (WHO, 2018). Sexual abuse questions were removed due to the potential risk of stigmatizing the respondents at their marital homes. To discern the impacts of different ACE subtypes and methods of representation, we created a dichotomized score indicating any exposure to ACEs, a summed score, a categorical variable $(0,1,2,3,4+\mathrm{ACEs})$, and indicators for the theorized domains: neglect, family psychological distress, home violence, and community violence (Table 1). The domains are dichotomous, representing exposure to any of the ACEs comprising the domain.

Perceived stress. Perceived stress was measured using the Perceived Stress Scale (PSS). The PSS is a 10 item instrument that measures the degree to which life situations are appraised as stressful in the last month (28). The summed score ranges from 0 to 40 and has demonstrated reliability and validity in South Asia $(29,30)$.

Anxiety. Anxiety was measured using the seven-item version of the General Anxiety Disoder screening tool (GAD7). The frequency of symptoms in the last two weeks is assessed on a four point Likert scale. The summed score ranges from 0 to 21 , and a cut-off of 10 typically represents a clinically significant level to identify probable cases of genaeraliesd anxiety disorder (31). The total summed GAD score and the dichotomized GAD indicator (a score above 10) were both used as outcomes in analyses. This measure has demonstrated reliability and validity in Pakistan (32).

Cortisol. Hair samples were collected using a standard protocol: approximately 200 strands of hair were cut from the posterior vertex. Research suggests that one centimeter of hair reflects hormonal output for the previous month (33). The present analysis utilizes two centimeters of hair, reflecting HPA-axis hormone output for the past two months. Cortisol was extracted and measured by Dresden LabService using standard liquid chromatography mass spectrometry $(34,35)$. We did not observe any outliers. Levels are reported as pg/mg.

Covariates. Informed by existing literature, several variables were selected for the models to adjust for design characteristics and to identify potential confounders. These included maternal age, natal family's history of mental illness, maternal education (as an indicator of childhood SES), baseline depression, peer-delivered perinatal intervention allocation status, and assessor at 36 months (36).

\section{Statistical Analysis}

For this cross-sectional analysis, a series of generalized linear models with an identity link were created to estimate coeffiecients for the total PSS and GAD scores as well for the maternal hair cortisol concentrations. Prevalence ratios for the dichotomized GAD were generated through log-Poisson models. All models used clusterrobust standard errors to account for clustering at the village cluster level. The PSS and GAD models were adjusted for baseline depression and treatment allocation status, mother's age at baseline and education level, history of natal family mental health, and assessor at 36 months.For each outcome (perceived stress, GAD, and hair cortisol), we examined associations with: 1) the presence of any ACEs (yes/no); 2) the number of endorsed ACEs modeled as a continuous indicator; 3 ) the number of endorsed ACEs modeled as one categorical variable 
with levels (i.e., 0, 1, 2, 3, 4+ ACEs); and 4) dichotomous inidcators for each domain of ACEs (neglect, family psychological distress, home violence, and community violence). Stata version 16.1 was used for data analysis (StataCorp, College Station, TX).

\section{Results}

\section{Descriptive Statistics}

Our analytic sample consisted of 889 women who were interviewed at 36 months postpartum (see table 2 for full demographic information). The majority (66.4\%) received at least a fifth grade education and had one to three children (62.5\%); the mean age (standard deviation [SD]) at baseline was 26.7 (4.4) years old. About 10\% reported growing up in a household with a family number who had a mental health illness. The mean (SD) total score on the PSS-10 stress scale was 12.0 (8.8). Fifty-eight percent of women experienced at least one ACE (Table 3), with a mean (SD; minimum, maximum) total number of ACEs of $1.2(1.4 ; 0,10)$. Among the 12 specific ACE items, emotional abuse (33.1\%), physical abuse (23.5\%), and emotional neglect (15.1\%) were the most common. The most prevalent ACE domain was home violence (38.3\%), followed by neglect $(19.9 \%)$, family psychological distress (15.8\%), and community violence (6.6\%) (Table 3). The distribution of ACEs were similar across the GAD and cortisol sub-samples.

Of the 623 women present at the 36 month wave that were able to complete the GAD-7, the mean (SD) total score was 4.0 (5.4), and 101 of the 623 participants (16.2\%) had a score above the clinically significant cut-off of 10 , which indicates moderate anxiety and signifies reasonable identification of GAD cases. A subset of 90 women participated in hair cortisol collection. There were no clear differences between the analytic groups $(\mathrm{N}=889$ for the PSS group, $\mathrm{N}=623$ for the GAD group, and $\mathrm{N}=90$ for the cortisol group) on demographic characteristics. The only distinctions were that women in the GAD sample tended to be younger, and the mean number of ACEs was higher in the cortisol subsample.

\section{Perceived Stress}

\section{Anxiety}

Both the presence of any ACEs and the continuous total ACE score were associated with higher anxiety symptoms on the GAD-7 at 36 months postpartum (Table 4, panel 2, models 1 and 2, continuous score $B=0.4$; $95 \% \mathrm{Cl}=0.04,0.8$ and dichotomous $\mathrm{ACE} \mathrm{B}=1.1 ; 95 \% \mathrm{Cl}=0.1,2.0)$. As compared to the perceived stress (PSS), there was less evidence of a dose-response relationship between the discrete count of ACEs and anxiety symptoms (model 3). Of all the domains, the strongest association existed with the history of family psychological distress $(\mathrm{B}=1.6 ; 95 \% \mathrm{Cl}=0.3,2.9)$.

Total ACE score was not related to the dichotomized GAD-7 (score > 10) (panel 3). However, those with a history of family distress experienced a slightly elevated prevalence of anxiety symptoms on GAD-7 (PR=1.6; 95\% Cl=1.0, 2.3). Mothers who experienced neglect had a lower prevalence of experiencing clinically significant anxiety symptoms ( $\mathrm{PR}=0.2 ; 95 \% \mathrm{Cl}=0.05,0.8)$.

\section{Cortisol}


The effect estimates among the cortisol subsample were generally in the direction of higher ACEs being correlated with higher cortisol levels (Table 5). Of the specific ACEs domains, home violence $(B=6.7 ; 95 \% \mathrm{Cl}=2.7$, $10.8)$ and community violence $(B=7.5 ; 95 \% \mathrm{Cl}=1.4,13.6)$ were associated with increased hair cortisol.

\section{Discussion}

The purpose of this study was to examine the impact of ACEs on perceived stress, anxiety symptoms, and cortisol in a sample of mothers residing in rural Pakistan. Our results revealed that ACEs are associated with increased perceived stress and anxiety later in adult life. These findings contribute to previous work indicating that the number of ACEs an individual experiences predicts their psychological functioning in adulthood (10, 11, 37). When examining perceived stress, we found that most ACE indicators, including the continuous measure of ACEs, the dichotomous indicators for number of ACEs, and specific ACEs domains, predicted the outcome in a similar way, with the exception of neglect. Similarly, for anxiety symptoms, there was a significant association of the total ACE score, but not the individual indicators of number of ACEs or ACEs domains. Together, these results suggest that the cumulative effect of multidimensional early adversity, as captured by a continuous ACE measure, may be more important in predicting adult stress than the individual domains of adversity or individual indicators of ACEs.

In this sample, ACEs were consistently and strongly associated with perceived stress, and somewhat more weakly associated with the continuous version of the anxiety symptom scale, and weakly associated when dichotomized at clinically significant levels. The PSS captures one's appraisal of situations as overwhelming or stressful, beyond the actual content of situations $(38,39)$. Our findings suggests that those who have ACEs are more likely to feel overwhelmed with any current conflict or instability $(4,40)$. Furthermore, accumulaton of disadvantges (i.e., a higher number of ACEs) increases the likelihood that individuals who had ACEs end up in a stressful environment later in life, potentially explaining the predictive power of ACEs on perceived stress (41).

Complementing the perceived stress measure, the assessment of generalized anxiety symptoms with the GAD-7 captures the experience of chronic worry and physiological dysregulation (e.g., muscle tension, restlessness) that are not explicitly tied to current stressors present in the environment. The focus is more on specific symptoms and less on the appraisal and subjective aspects of those symptoms, which is central to perceived stress $(28,31)$. Our findings with anxiety symptoms were not as consistent as with perceived stress, as can be seen in the finding that ACEs are associated with the continuous anxiety symptom count but not with anxiety at the dichotomized clinically meaningful level. This is consistent with the idea that ACEs might more strongly impact how adults appraise potentially stressful experiences as opposed to their body's physiological responses to those experiences (42). This could also be due to differences in measurement between a continuous score and a dichotomous cut-off.

Somewhat surprisingly, we also found that those who reported experiencing neglect as children were less likely to report significant anxiety symptoms. This is contrary to previous literature suggesting that neglect is associated with heightened anxiety symptoms, though neglect also behaved strangely with perceived stress (ie: neglect was negatively associated with perceived stress) $(43,44)$. This result may be traced to mothers who have experienced neglect being less likely to report anxiety symptoms independent of their actual experience of anxiety due to desensitization to deprivation-related experiences that tend to be more frequent, yet individually less severe than threat-related ones $(40,45,46)$. While disentangeling this finding is beyond the scope of this 
paper, desensitization and normalization mechanisms should be further explored. In Southeast Asian and subSaharan African studies, early adversity in the form of neglect was linked to emotional difficulties later in life (47). Additionally, neglect was not significantly associated with the continuous GAD-7 score, pointing to different relationships between neglect and clinically significant anxiety versus the continuous GAD-7.

When examining the association between ACEs and hair cortisol, we found that endorsing two ACEs or experiencing home violence were significant predictors of increased hair cortisol. This partially aligned with our hypothesis that early adversity is associated with increased HPA axis hyperactivity, and subsequent increase in cortisol production $(14,48)$. Interestingly, a study in India analyzed hair cortisol in children and found it to be linked with adversity (49). Other studies have shown that among early life experiences, violence particularly has a potent impact on programming the HPA axis (50). This is preliminary evidence that ACEs have downstream effects of adulthood HPA axis functioning. Future studies would benefit from a larger sample size to more fully investigate the relationship between ACEs exposure and cortisol.

In LMIC, home violence in the form of interpersonal violence is particularly common, which is also reflected in our sample $(51,52)$. Home violence was the only domain that significantly predicted cortisol, possibly because it is linked to consequences that are emotional in nature: isolation, fear, guilt, low self-esteem (53). Violence experienced at home may be internalized to a greater extent because it is physical, occuring closer to the child with a large presence in the child's life. Domestic violence also increases the risk for other kinds of abuse (54). Young girls living in households with domestic violence are are at risk for developing internalizing mental health issues (55). Importantly, we did not control for current interpersonal violence, which might interact with earlier adverse exposures and mediate HPA axis activity. We also did not find associations between the emotion abuse items and cortisol. Threatening, violent behaviors lead to the activated kinds of outcomes we focused on compared to ones that stem from neglectful experiences, which tend to be emotional in nature (56).

This study benefited from a number of strengths. In particular, we investigated the impact of ACEs in many ways: as individual items, categorically, continuously, binary (any/none), and domains (neglect, home violence, family psychological distress, community violence). In particular, few prior studies have examined childhood adversity in the form of neglect (45). Neglect is the most common type of maltreatment, increasing the importance to explore it in research $(40,45)$. Thus, we broke neglect into its subtypes of emotional and physical. Neglect behaved differently from the other domains, and future studies should examine neglect separately in addition to investigating its impact alongside other types of ACEs. In addition to modeling ACEs in multiple ways, we also studied the outcomes from two angles--perceived stress and anxiety-to discern nuances between the two seemingly similar measures. Although PSS and GAD-7 appear to measure similar things, the results of our study point to their distinctive relationships with ACEs. Additionally, the inclusion of the cortisol subanalysis is another way to approach stress and anxiety, with cortisol representing a biological constrast to behavioral constructs, PSS and GAD-7. Finally, our study was situated in a LMIC context, where ACEs have been understudied yet pose a significant public health burden (57).

These results should be interpreted in the context of several limitations. First, the cortisol exploraratory analysis only included 90 women, potentially reducing the validity of the associations among ACEs and cortisol. Additionally, Another limitation is that the ACEs questionnaire involved recall bias due to questions asking about events that occurred years, if not decades ago. However, the ACEs questionare was designed to be asked retrospectively (World Health Organization, 2018). All of the measures were self-reported and focused on the 
appraisal of experiences rather than the actual objective content, providing another pathway to produce subjective results (42). ACEs have been found to be comorbid with low-income and low-education populations, which predispose people to stressful living environments. We attempted to address this by controlling for maternal education as an indicator of childhood socioeconomic status. However, we did not include current stressful life events and violence exposure.

These results inform our understanding of how ACEs are associated with perceived stress, anxiety, and HPA-axis functioning. ACEs were associated with heightened levels across all three domains of perceived stress, anxiety, and cortisol, with varying precision and strength of estimates. Our study attempts to disentangle adversity into subtypes (neglect, home violence, family psychological distress, community violence) to pinpoint the impact of specific adverse events on HPA axis functioning, and therefore mental health conditions related to stress. Most studies of ACEs have focused on higher-income, well-educated populations, yet toxic stress tends to be embedded in social disadvantage and intergenetational adversity is often comorbid with other forms of maltreatment (7). Future research should center on identifying potential moderators and mediators between ACEs and perceived stress and anxiety in order to create targeted interventions that support mental health among women who have experienced ACEs and reside in LMICs. For instance, social support, emotion regulation skills, and positive self-perceptions are powerful protective factors that buffer women from the harmful effects of ACEs. Interventions targeting girls within community or school setting that harness these protective factors or prevent the consequences of ACEs have the potential to lessen HPA axis hyperactivity, prevent the intergenerational transmission of adversity, and improve mental health outcomes in this population.

\section{Abbreviations}

ACEs: Adverse childhood experiences; HPA axis: Hyothalamic pituitary adrenal axis; LMIC: Low- and middleincome countries; PSS: Perceived stress scale; GAD-7: Generalized anxiety disorder scale; SES: Socioeconomic status; SD: Standard deviation

\section{Declarations}

\section{Patient and Public Involvement}

Patient collaborators were not directly involved in the design, recruitment, or dissemination of this study. However, women in the recruitment populations have expressed a high degree of interest in the issue of maternal mental health.

\section{Ethics Approval and Consent to Participate}

The study was approved by institutional review boards at the Human Development Research Foundation, Duke University, and the University of North Carolina at Chapel Hill.

\section{Consent for Publication}

Not applicable.

\section{Availability of Data and Materials}


The datasets used in the current study are available from the corresponding author on reasonable request.

\section{Competing Interests}

The authors declare that they have no competing interests.

\section{Role of Funding Source}

The study and study team received support from the U.S. Eunice Kennedy Shriver National Institute of Child Health and Human Development for the larger research program (R01HD075875) and Robertson Scholars Leadership Program for general support and the U.S. National Institute of Mental Health for research program funding (U19MH95687). Hair hormone collection and analysis was funded by the University of Melbourne. Funding for analyses was further secured from the NICHD (R03HD097434). JAG was supported in part by CTSA grant UL1TR002553.

\section{Author Contributions}

NI, JM, AF, ES conceptualized the analysis. NI and VB analyzed the data and JAG, AF, JM further contributed to the data interpretation. NI drafted manuscript and all authors contributed substantively to revisions and further edits. JM, AH, VB, SS designed the larger study. All authors approved the final version to be submitted for publication, and $\mathrm{NI}$ was responsible for final submission.

\section{Acknowledgements}

The authors thank the team at the Human Development Research Foundation (HDRF), including Rakshanda Liaqat, Tayyiba Abbasi, Maria Sharif, Samina Bilal, Quratul-Ain, Anum Nisar, Amina Bibi, Shaffaq Zuqar, Sonia Khan, Ahmed Zaidi, Ikhlaq Ahmad, and Najia Atif for their contributions to the study's data collection, design and implementation. We also want to acknowledge the larger Bachpan and SHARE CHILD study teams as well as the women, children, and communities that are a part of the Bachpan cohort. We are also grateful to Professor Fareed Aslam Minhas from the Institute of Psychiatry, Rawalpindi Medical University for the assistance in the biomarker component of the study.

\section{References}

1. Hughes K, Bellis MA, Hardcastle KA, Sethi D, Butchart A, Mikton C, et al. The effect of multiple adverse childhood experiences on health: a systematic review and meta-analysis. The Lancet Public Health. 2017;2(8):e356-e66.

2. De Venter M, Demyttenaere K, Bruffaerts R. The relationship between adverse childhood experiences and mental health in adulthood. A systematic literature review. Tijdschrift voor psychiatrie. 2013;55(4):259-68.

3. McLafferty M, Armour C, McKenna A, O’Neill S, Murphy S, Bunting B. Childhood adversity profiles and adult psychopathology in a representative Northern Ireland study. Journal of Anxiety Disorders. 2015;35:42-8.

4. Manyema M, Norris SA, Richter LM. Stress begets stress: The association of adverse childhood experiences with psychological distress in the presence of adult life stress. BMC public health. 2018;18(1):1-12.

5. Fisher J, Mello MCd, Patel V, Rahman A, Tran T, Holton S, et al. Prevalence and determinants of common perinatal mental disorders in women in low-and lower-middle-income countries: a systematic review. Bulletin 
of the World Health Organization. 2012;90:139-49.

6. Walker SP, Wachs TD, Grantham-McGregor S, Black MM, Nelson CA, Huffman SL, et al. Inequality in early childhood: risk and protective factors for early child development. The lancet. 2011;378(9799):1325-38.

7. Rieder AD, Roth SL, Musyimi C, Ndetei D, Sassi RB, Mutiso V, et al. Impact of maternal adverse childhood experiences on child socioemotional function in rural Kenya: Mediating role of maternal mental health. Developmental science. 2019;22(5):e12833.

8. Ben-Shlomo Y, Kuh D. A life course approach to chronic disease epidemiology: conceptual models, empirical challenges and interdisciplinary perspectives. Oxford University Press; 2002.

9. Irish L, Kobayashi I, Delahanty DL. Long-term physical health consequences of childhood sexual abuse: A meta-analytic review. Journal of pediatric psychology. 2010;35(5):450-61.

10. Mersky J, Topitzes J, Reynolds AJ. Impacts of adverse childhood experiences on health, mental health, and substance use in early adulthood: A cohort study of an urban, minority sample in the US. Child abuse \& neglect. 2013;37(11):917-25.

11. Seiler A, Kohler S, Ruf-Leuschner M, Landolt MA. Adverse childhood experiences, mental health, and quality of life of Chilean girls placed in foster care: An exploratory study. Psychological trauma: theory, research, practice, and policy. 2016;8(2):180.

12. Negriff S. ACEs are not equal: Examining the relative impact of household dysfunction versus childhood maltreatment on mental health in adolescence. Social Science \& Medicine. 2020;245:112696.

13. LeMasters K, Bates LM, Chung EO, Gallis JA, Hagaman A, Scherer E, et al. Adverse childhood experiences and depression among women in rural Pakistan. BMC public health. 2021;21(1):1-11.

14. Danese A, McEwen BS. Adverse childhood experiences, allostasis, allostatic load, and age-related disease. Physiology \& behavior. 2012;106(1):29-39.

15. Hagaman AK, Baranov V, Chung E, LeMasters K, Andrabi N, Bates LM, et al. Association of maternal depression and home adversities with infant hypothalamic-pituitary-adrenal (HPA) axis biomarkers in rural Pakistan. Journal of Affective Disorders. 2020;276:592-9.

16. Nurius PS, Green S, Logan-Greene P, Longhi D, Song C. Stress pathways to health inequalities: embedding ACEs within social and behavioral contexts. International public health journal. 2016;8(2):241.

17. Khoury JE, Enlow MB, Plamondon A, Lyons-Ruth K. The association between adversity and hair cortisol levels in humans: A meta-analysis. Psychoneuroendocrinology. 2019;103:104-17.

18. Kalmakis KA, Meyer JS, Chiodo L, Leung K. Adverse childhood experiences and chronic hypothalamicpituitary-adrenal activity. Stress. 2015;18(4):446-50.

19. lob E, Lacey R, Steptoe A. The long-term association of adverse childhood experiences with C-reactive protein and hair cortisol: Cumulative risk versus dimensions of adversity. Brain, behavior, and immunity. 2020;87:318-28.

20. Naveed S, Waqas A, Chaudhary AMD, Kumar S, Abbas N, Amin R, et al. Prevalence of common mental disorders in South Asia: a systematic review and meta-regression analysis. Frontiers in psychiatry. 2020;11:899.

21. Chapman DP, Whitfield CL, Felitti VJ, Dube SR, Edwards VJ, Anda RF. Adverse childhood experiences and the risk of depressive disorders in adulthood. Journal of affective disorders. 2004;82(2):217-25. 
22. D'Anna-Hernandez KL, Ross RG, Natvig CL, Laudenslager ML. Hair cortisol levels as a retrospective marker of hypothalamic-pituitary axis activity throughout pregnancy: comparison to salivary cortisol. Physiology \& behavior. 2011;104(2):348-53.

23. Bhandari S, Levitch AH, Ellis KK, Ball K, Everett K, Geden E, et al. Comparative analyses of stressors experienced by rural low-income pregnant women experiencing intimate partner violence and those who are not. Journal of Obstetric, Gynecologic \& Neonatal Nursing. 2008;37(4):492-501.

24. Tariq S, Jawed S. Perceived stress, severity and sources of stress among female medical students in a private medical college in Pakistan. JPMA. 2020;2019.

25. Shah M, Hasan S, Malik S, Sreeramareddy CT. Perceived stress, sources and severity of stress among medical undergraduates in a Pakistani medical school. BMC medical education. 2010;10(1):1-8.

26. Shamsi U, Hatcher J, Shamsi A, Zuberi N, Qadri Z, Saleem S. A multicentre matched case control study of risk factors for preeclampsia in healthy women in Pakistan. BMC women's health. 2010;10(1):1-7.

27. Turner EL, Sikander S, Bangash O, Zaidi A, Bates L, Gallis J, et al. The effectiveness of the peer delivered Thinking Healthy Plus (THPP+) Programme for maternal depression and child socio-emotional development in Pakistan: study protocol for a three-year cluster randomized controlled trial. Trials. 2016;17(1):1-11.

28. Cohen S, Kamarck T, Mermelstein R. Perceived stress scale. Measuring stress: A guide for health and social scientists. 1994;10:1-2.

29. Chakraborti A, Ray P, Sanyal D, Thakurta RG, Bhattacharayya AK, Mallick AK, et al. Assessing perceived stress in medical personnel: in search of an appropriate scale for the Bengali population. Indian journal of psychological medicine. 2013;35(1):29.

30. Stubbs B, Veronese N, Vancampfort D, Prina AM, Lin P-Y, Tseng P-T, et al. Perceived stress and smoking across 41 countries: A global perspective across Europe, Africa, Asia and the Americas. Scientific reports. 2017;7(1):1-8.

31. Spitzer RL, Kroenke K, Williams JB, Löwe B. A brief measure for assessing generalized anxiety disorder: the GAD-7. Archives of internal medicine. 2006;166(10):1092-7.

32. Ahmad S, Hussain S, Shah FS, Akhtar F. Urdu translation and validation of GAD-7: A screening and rating tool for anxiety symptoms in primary health care. J Pak Med Assoc. 2017;67(10):1536140.

33. Stalder T, Kirschbaum C. Analysis of cortisol in hair-state of the art and future directions. Brain, behavior, and immunity. 2012;26(7):1019-29.

34. Gao W, Stalder T, Foley P, Rauh M, Deng H, Kirschbaum C. Quantitative analysis of steroid hormones in human hair using a column-switching LC-APCI-MS/MS assay. Journal of chromatography B, Analytical technologies in the biomedical and life sciences. 2013;928:1-8.

35. Gao W, Stalder T, Foley P, Rauh M, Deng H, Kirschbaum C. Quantitative analysis of steroid hormones in human hair using a column-switching LC-APCl-MS/MS assay. Journal of Chromatography B. 2013;928:18.

36. Cohen S, Janicki-Deverts D, Chen E, Matthews KA. Childhood socioeconomic status and adult health. Annals of the New York Academy of Sciences. 2010;1186(1):37-55.

37. Herrenkohl TI, Hong S, Klika JB, HerrenkohI RC, Russo MJ. Developmental impacts of child abuse and neglect related to adult mental health, substance use, and physical health. Journal of family violence. 2013;28(2):191-9. 
38. Katsarou AL, Triposkiadis F, Panagiotakos D. Perceived stress and vascular disease: where are we now? Angiology. 2013;64(7):529-34.

39. Hughes AJ, Dunn KM, Chaffee T, Bhattarai JJ, Beier M. Diagnostic and clinical utility of the GAD-2 for screening anxiety symptoms in individuals with multiple sclerosis. Archives of physical medicine and rehabilitation. 2018;99(10):2045-9.

40. Jackson AM, Kissoon N, Greene C. Aspects of abuse: recognizing and responding to child maltreatment. Curr Probl Pediatr Adolesc Health Care. 2015;45(3):58-70.

41. Rutter M. Pathways from childhood to adult life. Journal of child psychology and psychiatry. 1989;30(1):2351.

42. Danese A, Widom CS. Objective and subjective experiences of child maltreatment and their relationships with psychopathology. Nature human behaviour. 2020:1-8.

43. Spertus IL, Yehuda R, Wong CM, Halligan S, Seremetis SV. Childhood emotional abuse and neglect as predictors of psychological and physical symptoms in women presenting to a primary care practice. Child Abuse Negl. 2003;27(11):1247-58.

44. Cohen JR, Menon SV, Shorey RC, Le VD, Temple JR. The distal consequences of physical and emotional neglect in emerging adults: A person-centered, multi-wave, longitudinal study. Child Abuse Negl. 2017;63:151-61.

45. Ben-David V, Jonson-Reid M. Resilience among adult survivors of childhood neglect: A missing piece in the resilience literature. Children and youth services review. 2017;78:93-103.

46. McLaughlin KA, Sheridan MA, Lambert HK. Childhood adversity and neural development: deprivation and threat as distinct dimensions of early experience. Neuroscience \& Biobehavioral Reviews. 2014;47:578-91.

47. Escueta M, Whetten K, Ostermann J, O’Donnell K, Team POfOR. Adverse childhood experiences, psychosocial well-being and cognitive development among orphans and abandoned children in five low income countries. BMC international health and human rights. 2014;14(1):6.

48. Shenk CE, Putnam FW, Noll JG. Experiential avoidance and the relationship between child maltreatment and PTSD symptoms: Preliminary evidence. Child Abuse \& Neglect. 2012;36(2):118-26.

49. Bhopal S, Verma D, Roy R, Soremekun S, Kumar D, Bristow M, et al. The contribution of childhood adversity to cortisol measures of early life stress amongst infants in rural India: Findings from the early life stress substudy of the SPRING cluster randomised controlled trial (SPRING-ELS). Psychoneuroendocrinology. 2019;107:241-50.

50. Dajani R, Hadfield K, van Uum S, Greff M, Panter-Brick C. Hair cortisol concentrations in war-affected adolescents: A prospective intervention trial. Psychoneuroendocrinology. 2018;89:138-46.

51. Daley D, McCauley M, van den Broek N. Interventions for women who report domestic violence during and after pregnancy in low-and middle-income countries: a systematic literature review. BMC pregnancy and childbirth. 2020;20(1):1-9.

52. Hagaman A, Gallis JA, Bhalotra S, Baranov V, Turner EL, Sikander S, et al. Psychosocial determinants of sustained maternal functional impairment: Longitudinal findings from a pregnancy-birth cohort study in rural Pakistan. PloS one. 2019;14(11):e0225163.

53. Herrenkohl TI, Sousa C, Tajima EA, Herrenkohl RC, Moylan CA. Intersection of child abuse and children's exposure to domestic violence. Trauma, Violence, \& Abuse. 2008;9(2):84-99. 
54. Appel AE, Holden GW. The co-occurrence of spouse and physical child abuse: a review and appraisal. Journal of family psychology. 1998;12(4):578.

55. Widom CS. Childhood victimization: Early adversity and subsequent psychopathology. Adversity, stress, and psychopathology. 1998:81-95.

56. Sumner JA, Colich NL, Uddin M, Armstrong D, McLaughlin KA. Early experiences of threat, but not deprivation, are associated with accelerated biological aging in children and adolescents. Biological psychiatry. 2019;85(3):268-78.

57. Valdebenito S, Murray A, Hughes C, Băban A, Fernando AD, Madrid BJ, et al. Evidence for Better Lives Study: a comparative birth-cohort study on child exposure to violence and other adversities in eight low-and middleincome countries-foundational research (study protocol). BMJ open. 2020;10(10):e034986.

\section{Tables}




\section{Table 1. ACE International Domain and Question Definitions}

Description

Neglect

Emotional Neglect

Physical Neglect

\section{Family Psychological Distress}

Alcohol and/or drug abuser in household

Incarcerated household member

Someone chronically depressed, mentally ill, institutionalized, or suicidal

One or no parents, parental Parents ever separated/divorced, or father/mother/guardian died separation or divorce

\section{Home Violence}

Physical Abuse

You being spanked/ slapped/kicked/ punched/beaten up; or being hit/cut with an object by parents/guardian

Emotional Abuse

You were yelled at/screamed at/sworn at/insulted or humiliated, or were threatened/abandoned, thrown out by parents/guardian

Household member treated violently

Saw or heard a parent/household member being yelled at/screamed at/sworn at/ insulted or humiliated; being slapped/kicked/punched/beaten up; or being hit/cut with an object

\section{Community Violence}

Bullying

Community Violence

Collective Violence

\section{Being bullied}

See or hear someone being beaten up, stabbed, shot, or being threatened with a knife or gun in real life

Forced to go and live in other place; experienced disruption of home due to these 


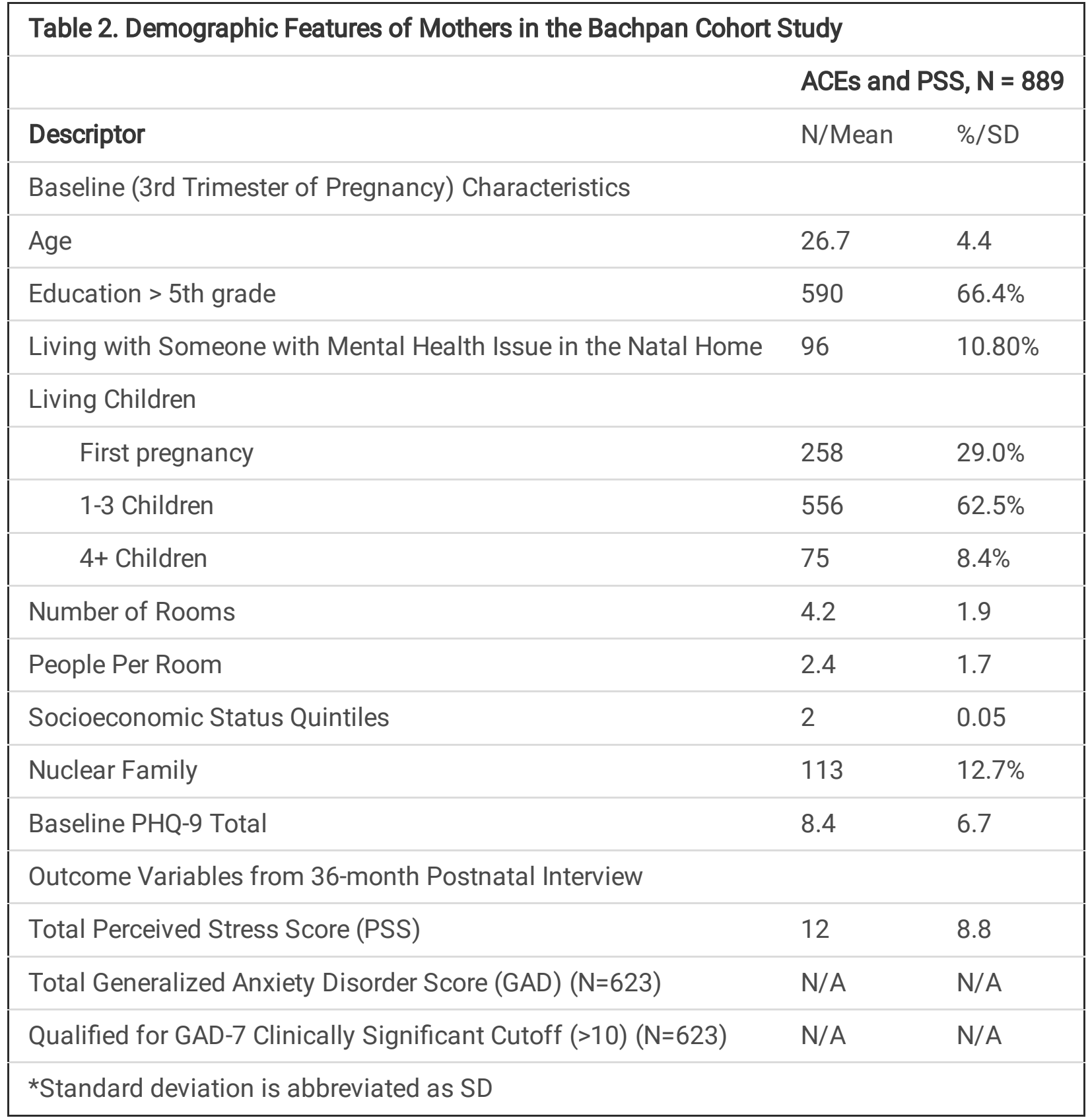




\section{Table 3. Maternal ACE Characteristics in Full Sample}

Description
Neglect

1. Emotional Neglect

134

$15.1 \%$

2. Physical Neglect

49

$5.5 \%$

\section{Family Psychological Distress}

3. Household alcohol and/or drug use

24

$2.7 \%$

4. Incarcerated household member

16

$1.8 \%$

5. Depressed, mentally ill, institutionalized, or suicidal household member 21

$2.4 \%$

6. One or no parents, parental separation, or divorce

97

$10.9 \%$

Home Violence

7. Physical Abuse

209

$23.5 \%$

8. Emotional Abuse

294

$33.1 \%$

9. Household member treated violently

130

$14.6 \%$

\section{Community Violence}

10. Bullying

12

$1.4 \%$

11. Community Violence

60

$6.8 \%$

12. Collective Violence

4

$0.5 \%$

\section{Domains}

Neglect

173

$19.9 \%$

Family Psychological Distress

143

$15.8 \%$

Home Violence

349

$38.3 \%$

Community Violence

64

$6.6 \%$

Number of ACEs

\begin{tabular}{|lcc|}
\hline ACEs & 369 & $41.5 \%$ \\
\hline 1 ACE & 239 & $26.8 \%$ \\
\hline 3 ACEs & 139 & $15.6 \%$ \\
\hline $4+$ ACEs & 82 & $9.2 \%$ \\
\hline Any ACE Exposure & 61 & $6.8 \%$ \\
\hline
\end{tabular}




\section{Table 4. Maternal ACEs and Perceived Stress and Anxiety at 36 Months \\ Postpartum}

\begin{tabular}{|c|c|c|c|c|c|c|c|c|c|c|}
\hline \multirow{4}{*}{$\begin{array}{l}\text { Model } \\
1\end{array}$} & \multirow{4}{*}{$\begin{array}{l}\text { Predictor } \\
\text { ACE Total }\end{array}$} & \multirow{2}{*}{\multicolumn{3}{|c|}{$\begin{array}{l}\text { Panel } 1 \\
\text { Stress (PSS), } \mathbf{N}=\mathbf{8} 89\end{array}$}} & \multirow{2}{*}{\multicolumn{3}{|c|}{$\begin{array}{l}\text { Panel } 2 \\
\text { Anxiety (GAD-7), } N= \\
623\end{array}$}} & \multirow{2}{*}{\multicolumn{3}{|c|}{$\begin{array}{l}\text { Panel } 3 \\
\text { GAD Dichotomized at } \\
\text { Cutoff of } 10, N=623\end{array}$}} \\
\hline & & & & & & & & & & \\
\hline & & \multirow{2}{*}{$\begin{array}{l}\text { B } \\
1.0\end{array}$} & \multicolumn{2}{|c|}{$95 \% \mathrm{Cl}$} & \multirow{2}{*}{ B } & \multicolumn{2}{|c|}{$95 \% \mathrm{Cl}$} & \multirow{2}{*}{$\begin{array}{ll}P R \\
1.0\end{array}$} & \multicolumn{2}{|c|}{$95 \% \mathrm{Cl}$} \\
\hline & & & 0.7 & 1.3 & & 0.04 & 0.8 & & 0.8 & 1.1 \\
\hline 2 & Any ACE & 2.4 & 1.0 & 3.8 & 1.1 & 0.1 & 2.0 & 1.1 & 0.7 & 1.6 \\
\hline \multirow[t]{5}{*}{3} & 0 ACEs & Ref & & & Ref & & & Ref & & \\
\hline & $1 \mathrm{ACE}$ & 2.1 & 0.3 & 4.0 & 0.9 & -0.3 & 2.2 & 1.2 & 0.7 & 2.0 \\
\hline & 2 ACEs & 1.2 & -0.6 & 3.0 & 0.9 & -0.3 & 2.2 & 1.2 & 0.7 & 2.1 \\
\hline & 3 ACEs & 3.1 & 0.9 & 5.3 & 1.7 & 0.2 & 3.2 & 1.2 & 0.7 & 2.0 \\
\hline & 4+ ACEs & 5.5 & 3.7 & 7.3 & 1.2 & -0.7 & 3.2 & 0.4 & 0.1 & 1.5 \\
\hline 4 & Neglect & 1.0 & -0.5 & 2.6 & -0.8 & -2.3 & 0.8 & 0.2 & 0.05 & 0.8 \\
\hline 5 & $\begin{array}{l}\text { Family } \\
\text { Psychological } \\
\text { Distress }\end{array}$ & 3.0 & 1.3 & 4.7 & 1.6 & 0.3 & 2.9 & 1.6 & 1.0 & 2.3 \\
\hline 6 & $\begin{array}{l}\text { Home } \\
\text { Violence }\end{array}$ & 1.6 & 0.6 & 2.7 & 0.7 & -0.2 & 1.5 & 1.0 & 0.7 & 1.4 \\
\hline 7 & $\begin{array}{l}\text { Community } \\
\text { Violence }\end{array}$ & 3.0 & 1.1 & 4.9 & 1.6 & -0.6 & 3.8 & 0.9 & 0.4 & 1.8 \\
\hline \multicolumn{11}{|c|}{$\begin{array}{l}\text { Note. Models take into account clustering at the village cluster level using cluster robust standard errors, an } \\
\text { models are adjusted for mother age at baseline, education level, natal family history of mental health, } \\
\text { baseline depression and treatment status, and assessor. }\end{array}$} \\
\hline
\end{tabular}




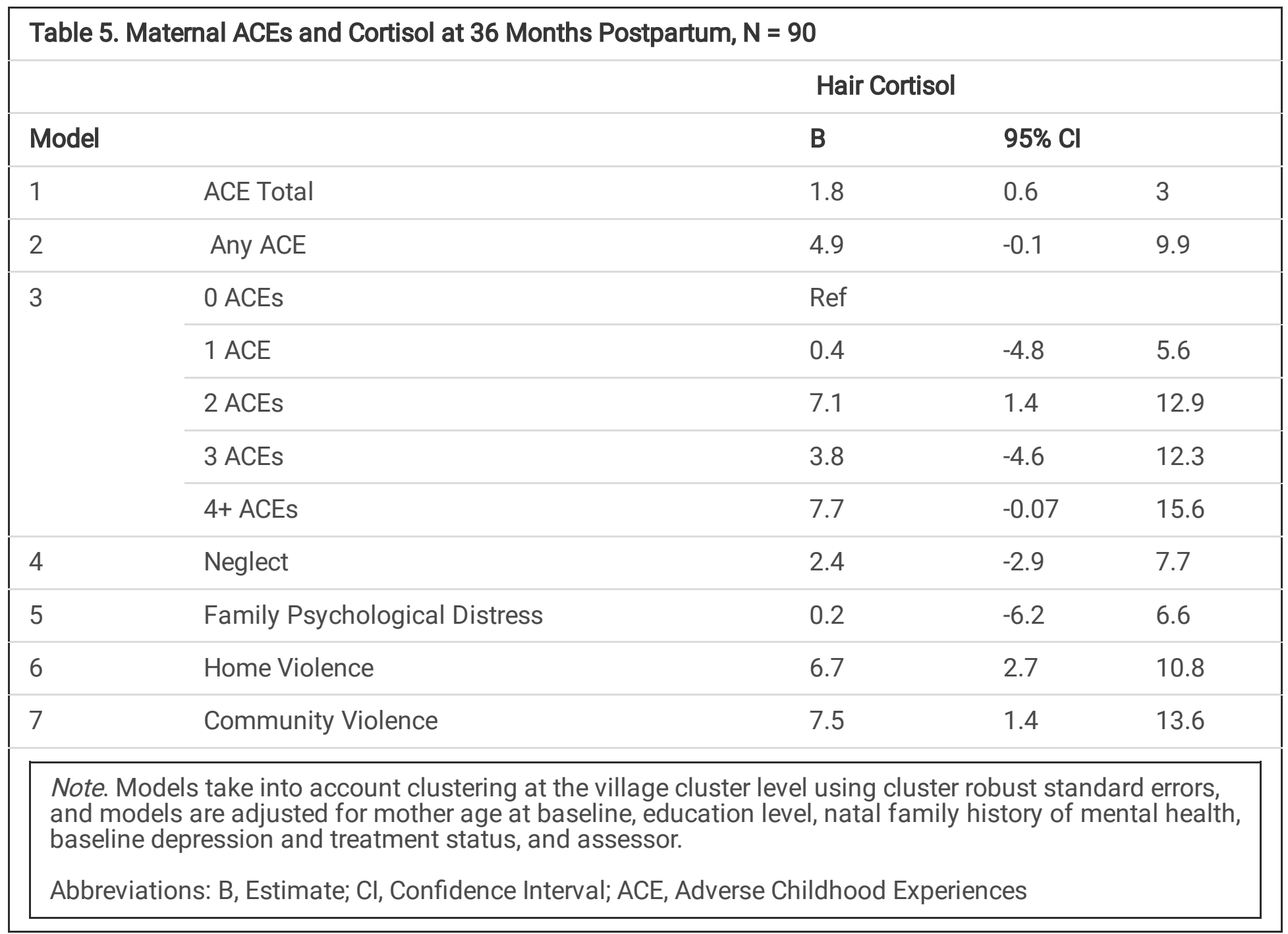

\section{Supplementary Files}

This is a list of supplementary files associated with this preprint. Click to download.

- ACEsPerceivedStressAnxietyCortisolTables.docx 